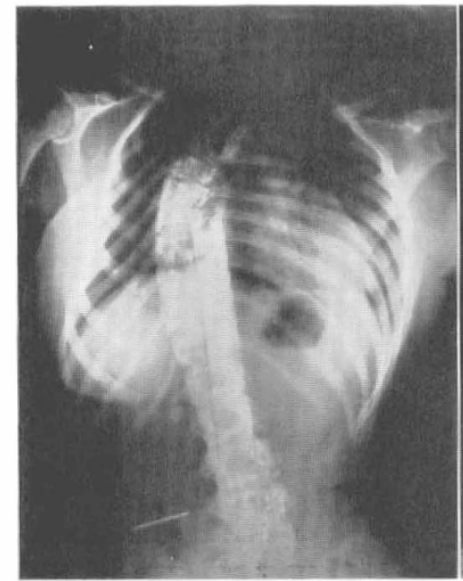

A

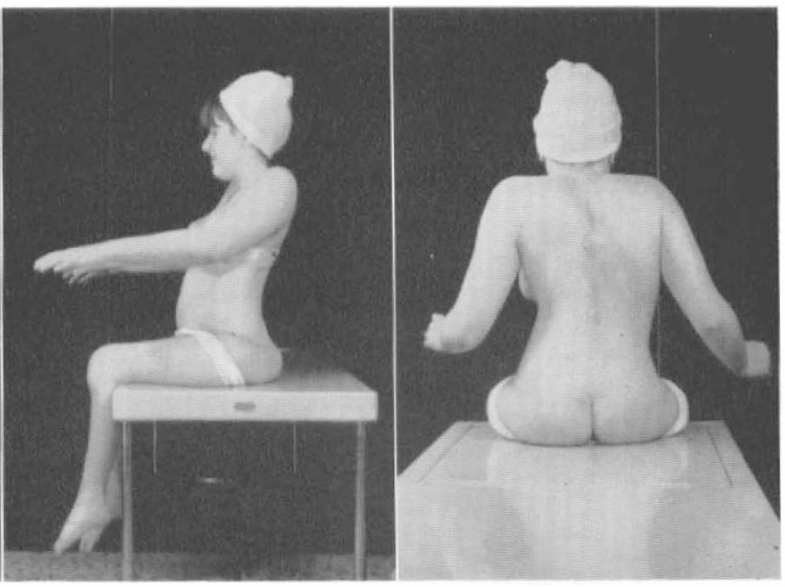

B
C

FIG. 6

A, B and C, Same patient three years after spinal grafting shows a most remarkable improvement of spinal deformity and posture.

a grotesque deformity of the spine and trunk with postural collapse, as shown in Figures 5, A, B and C. This patient underwent surgical treatment by spinal grafting and made a most remarkable improvement following this operation, as shown in Figure 6, A, B and $\mathrm{C}$.

\title{
EVALUATION OF LATE SPINAL DEFORMITIES WITH FRACTURE-DISLOCATIONS OF THE DORSAL AND LUMBAR SPINE IN PARAPLEGICS
}

\author{
John D. Leidholt, M.D. ${ }^{1}$, John J. Young, M.D. ${ }^{3}$, Harry R. Hahn, \\ M.D. ${ }^{2}$, Robert E. Jackson, M.D. ${ }^{2}$, William E. Gamble, M.D. ${ }^{\text {, }}$ \\ and James S. Miles, M.D. ${ }^{4}$
}

\section{PROBLEM AND INTRODUCTION}

ThE purpose of this clinical study was to determine which deformities of the dorsal and lumbar spine produced by fractures and dislocations were important. These deformities were to be considered in regard to return of spinal cord and nerve root function, as well as the effect of the deformities on sitting and walking as well as disabling back pain. Of special concern was the identification of deformities which proved to be progressive. On the basis of such a study, one would like to be able to predict which fracture dislocations of the spine should be relocated and which should be stabilized by fusion and by which operative method.

1 Denver Orthopedic and Fracture Clinic, Denver, Colorado, U.S.A.

${ }^{2}$ Craig Rehabilitation Hospital, Rocky Mountain Region Spinal Injuries Center, Denver, Colorado, U.S.A.

${ }^{3}$ Good Samaritan Hospital, Phoenix, Arizona, U.S.A.

${ }^{4}$ University of Colorado Medical Center, Denver, Colorado, U.S.A. 
The spin-off benefit of this special study might be some light on the problem of the indications for immediate post-injury laminectomy, as well as spine fusion.

This of course was a retrogressive study with all of the defects of such an analysis.

The experience at the Rocky Mountain Region Spinal Injury Centre in Denver has been such that we have had the opportunity to study patients treated by various means, operative and non-operative, referred from our region as well as many others. Many patients have been referred after definitive treatment such as laminectomy and fusion. Our care to the acute spinal injured patient has been to do a laminectomy only when specific indications were present and defer spine fusion. A post-laminectomy patient with a dorsal or lumbar fracture dislocation, even with displacement, has been treated with bed rest on a Stryker frame for six weeks, followed by sitting or standing in an appropriate brace, a Jewett or BrownNorton type. At the end of 12 weeks, bending films, lateral view of the spine in full flexion and extension, are obtained. If motion at the fracture site is noted, spine fusion or continued back support is recommended. In very few patients has operative treatment of the spine been indicated. It is with this clinical experience that the opportunity to study spine deformities with fracture dislocations presented itself. A need to reassess this non-operative approach for the care of these patients prompted this clinical study.

Furthermore, the interest in recent writings by Aufranc et al. (I968) regarding 'micro-instability' and the effect of spine deformity on cord and nerve root recovery also stimulated the authors. Also, we had previously drawn some general conclusions that the severity of the late spine deformity rarely affected the final result, either functionally in sitting or standing, or the recovery neurologically. In other words, the initial damage determined the final result, and the deformity rarely affected the final status of the patient. Detailed study of these paraplegic patients with emphasis on the back deformity has not been done and certainly was needed.

\section{METHOD}

This is a retrospective study of patients with traumatic paraplegia due to fracture-dislocations of the dorsal and lumbar spine admitted to the Rocky Mountain Region Spinal Injuries Center between I956 and 1967. Minimal followup period was one year, a maximum of ro years. This group of 204 patients did not include those spine injuries caused by penetrating wounds. Many were patients who had received their initial treatment elsewhere and were referred for late-stage treatment.

The method of study was to select from the entire group the patients with the most severe spinal deformities. In this group one would expect to find the complications of pain, poor function or pressure sores if spine deformity was a significant factor.

Selected from the entire group were the patients who had a kyphosis, or deformity in the AP plane of 30 degrees or more. Also included in the study were cases with a scoliosis or lateral angulation of Io degrees or more. The degree of deformity was arbitrarily chosen: there was no precedent for selection of these specific deformities. Some patients were included who had deformities in both planes, $\mathrm{AP}$ and lateral. All patients with deformities of a lesser degree were excluded.

Careful review of the medical records revealed that the patients tended to 
group into three general categories. The patients were divided into three groups: thoracic group, DI to Dio, thoraco-lumbar group, DII to LI; lumbar group, L2 to L5.

The curvatures were measured in degrees of deformity from a straight line using the two or three vertebrae at the fracture-dislocation site (see fig. I).

Following the selective separation of patients by X-ray, the medical records were reviewed.

Special attention was given to the presence of disabling back pain, neurological function return or loss, problems with regard to sitting, walking, and also skin breakdown problems.

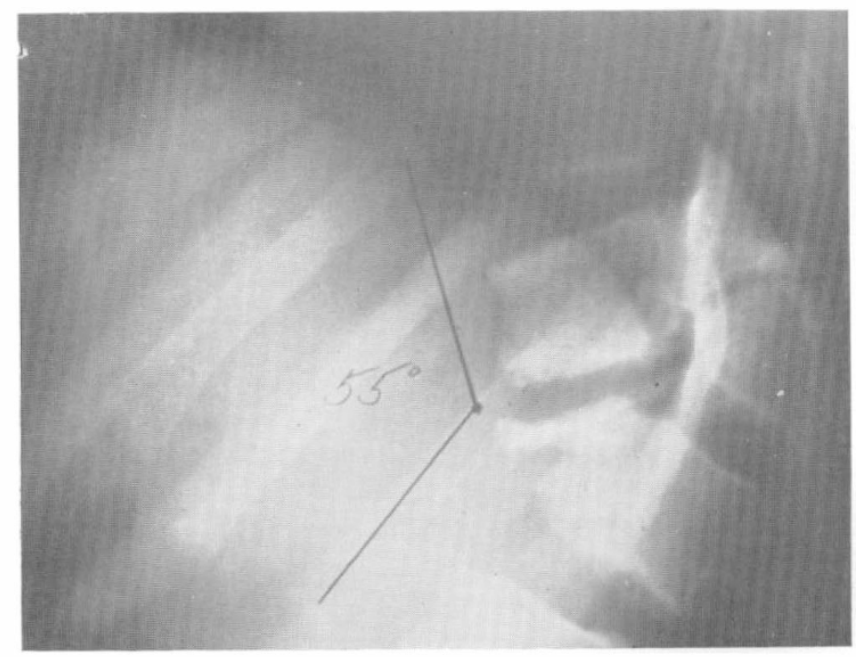

FIG. I

The method used for measuring the deformity in the AP plane is shown here. The anterior surface of an intact vertebra both above and below the fracture-dislocation complex was used to determine the angulation from a straight line. This method did not take into account normal degrees of kyphosis of the dorsal spine or lordosis of the lumbar spine.

\section{MATERIAL}

There were 204 patients with fracture-dislocation in the dorsal or lumbar spine with complete or incomplete neurological lesions admitted between 1956 and 1967. Fifty-eight out of the 204 patients had deformities either in the AP or lateral plane of sufficient degree to include them in their study.

Dorsal Group (DI-Dro) (25 patients). Twenty-five patients had A.P. deformities exceeding 30 degrees. All levels were involved from $\mathrm{D}_{3}$ to Dio.

Seven of the 25 AP deformities were at the D6 level, I 4 of the 25 were between D5 and D7.

Dorso-lumbar Group (DII-LI) (26 patients). This was the largest group in our series of dorso-lumbar spine injuries. There were 28 patients in this group with AP curves of more than Io degrees, and less than 30 degrees; these patients were not included in this study. 
Sixteen patients had AP curves of 30 degrees or more.

Six patients had AP deformities of 30 degrees or more with lateral deformities of Io degrees or more.

The average AP curve was 42 degrees.

Four patients had lateral curves of Io degrees or more, with $\mathrm{AP}$ curves of less than 30 degrees. lesions.

Sixty per cent. of this group had what was considered as complete neurological

Sixteen of the 26 at Diz level, three at Dir, and seven of the 26 were at the Lr level.

Lumbar Group (L2-L5) (7 patients). Four had AP curves exceeding 30 degrees.

One had AP curves of more then 30 degrees, and a lateral curve of Io degrees or more.

Two hac If Io degrees or more.

All of the AP curves of 30 degrees or more were at the level of L2.

The average AP curve was 52 degrees neurologically.

Seventy per cent. of the patients had complete cauda equina lesions.

TABLE

Summary of Spinal Deformities Studied

\begin{tabular}{|c|c|c|c|}
\hline & $\begin{array}{l}\text { AP curves of } 30 \\
\text { degrees or more }\end{array}$ & $\begin{array}{l}\text { AP of } 30 \text { degrees } \\
\text { or more with } \\
\text { lateral curve of Io } \\
\text { degrees or more }\end{array}$ & $\begin{array}{l}\text { Lateral deformity, } \\
\text { Io degrees or more }\end{array}$ \\
\hline Di-Dio & 25 & 0 & 0 \\
\hline DII-LI & I6 & 6 & 4 \\
\hline $\mathrm{L}_{2}-\mathrm{L} 5$ & 4 & I & 2 \\
\hline Total & 45 & 7 & 6 \\
\hline
\end{tabular}

\section{OBSERVATIONS}

Fifty-eight of 204 patients had what were considered as severe deformities after their spinal injuries. This of course meant that the patients had angular deformities that were considered significant. Some patients had displacement without angular deformity and were not included (fig. 2).

Fifty-two of 204 had AP deformities of 30 degrees or more.

Thirteen of 204 had lateral deformities of ro degrees or more.

\section{OBSERVATIONS IN GROUPS}

Dorsal Group DI-Dro. All fractures became solid spontaneously or with spine fusions.

No problems of balance in sitting or standing could be attributed to the deformity. 


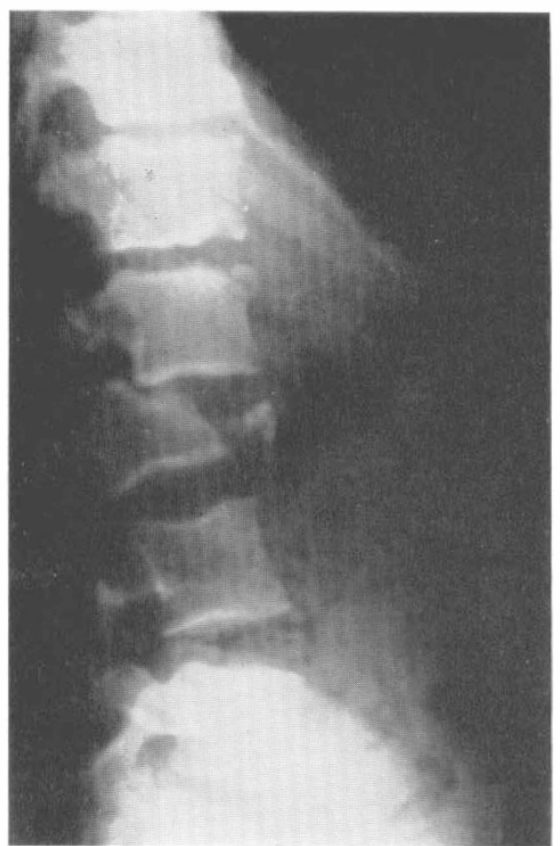

FIG. 2
Fig. 2.-In spite of the marked anterior displacement of $\mathrm{L}_{2}$ on $\mathrm{L}_{3}$, this patient was not included in the study until angulation occurred at a later date. Angular deformity was used as the parameter for selection, rather than displacement.

Fig. 3.-This kyphosis, although prominent due to a 45 degree anterior angulation of Dro, has not been a problem.

Fig. 4.-This patient's deformity progressed after the spine fusion to 46 degrees at LI before becoming solid. The angulation has not interfered with his standing or walking balance.

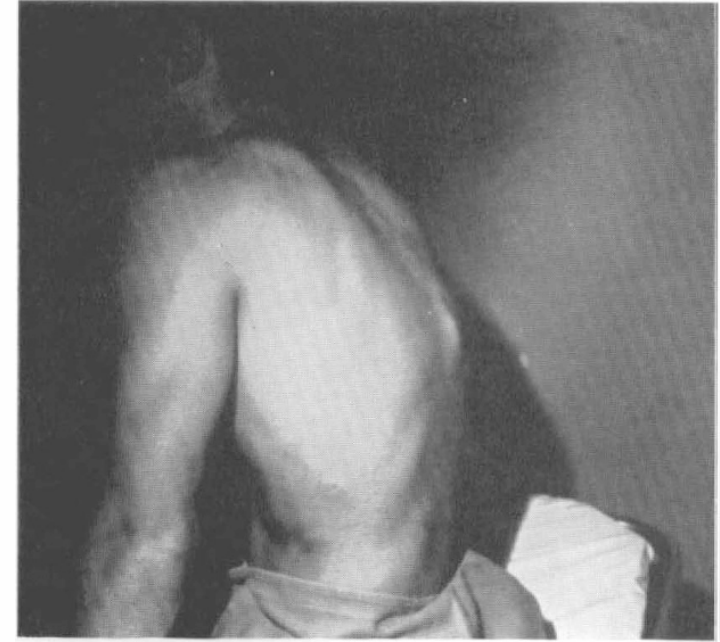

FIG. 3

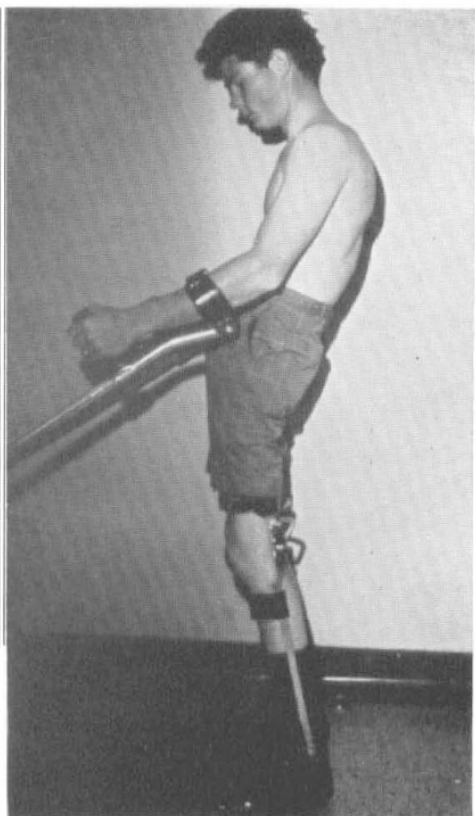

FIG. 4 
No altered recovery of cord function was attributed to the deformity.

Even the prominent kyphosis did not constitute a persistent pressure problem although special bracing design was needed (fig. 3).

Dorso-lumbar Group Di I-LI. Postural problems were limited to:

One patient with forward trunk stance with an $\mathrm{AP}$ curve at 45 degrees. heel lift.

One patient with a pelvic obliquity who had a stance problem requiring a

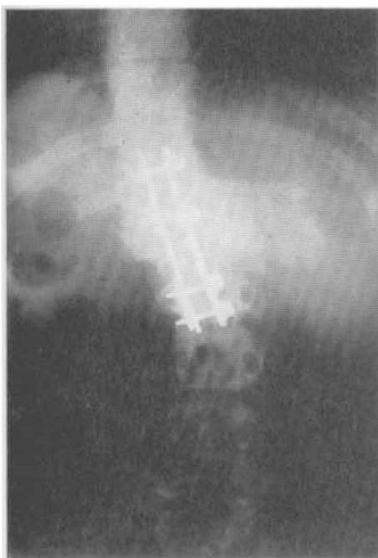

FIG. 5

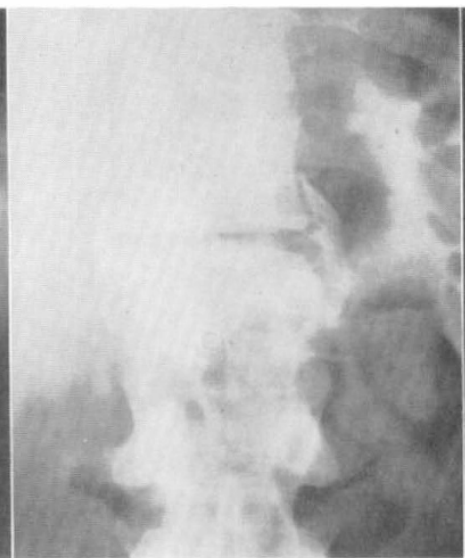

A

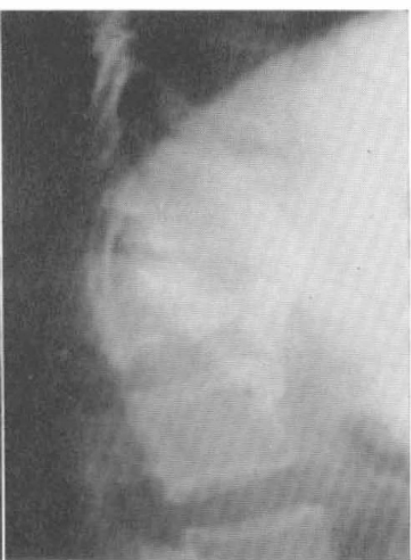

FIG. 6
B

Fig. 5.-This 24-year-old male was initially treated with a laminectomy and internal fixation with Harrington instruments. Because of failure to obtain a fusion, bone plates were used as well as autogenous bone grafts. The spine showed motion at the fracture site on bending films three years after the injury.

Eig. 6.-Twenty-two of 26 patients of the dorso-lumbar group demonstrated, on bending films, a fibrous or bone fixation at the fracture site. A typical example of spontaneous interbody fusion is seen in this illustration.

Progression of deformity occurred in two patients. These patients showed what appeared to be an increased density of the injured vertebral body which could be interpreted as aseptic or avascular necrosis. Bedbrook (I968) of Perth, Australia, has biopsy material to confirm this suspicion.

One of the two patients, a 2 I-year-old female who had a Di2 fracturedislocation with a complete neurological lesion showed progressive deformity. Spine fusion was advised but she refused the surgery. This spine became solid over a period of two years, but only after the deformity increased from 25 degrees to 50 degrees. She was having back pain at the end of this period. She had no limitation of function because of the back deformity. This patient had had a laminectomy, but no fusion.

Another young male 23 years of age, showed a progression of the Li deformity inspite of a laminectomy and an extensive fusion, $\mathrm{D}_{10}$ to $\mathrm{L}_{3}$, including adequate bone placed in the lateral gutters. Autogenous rib bone was used. He remained on a Stryker frame for three months. The spine fusion finally became solid, but only after the angular deformity reached 46 degrees. In spite of the deformity there have been no balance or pain problems after five years (fig. 4). 
Failure to achieve solid fusion with the use of internal fixation occurred in two patients. Harrington compression instruments were used in both. One patient continues to have back pain but this is not disabling after three years (fig. 5). All other spines were considered solid and showed no motion at the fracture site with bending X-rays (fig. 6).

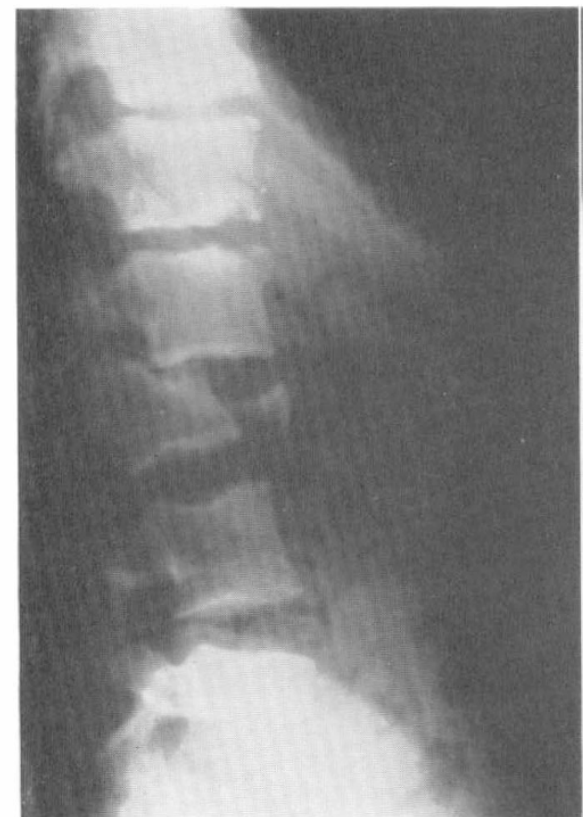

Fig. 7

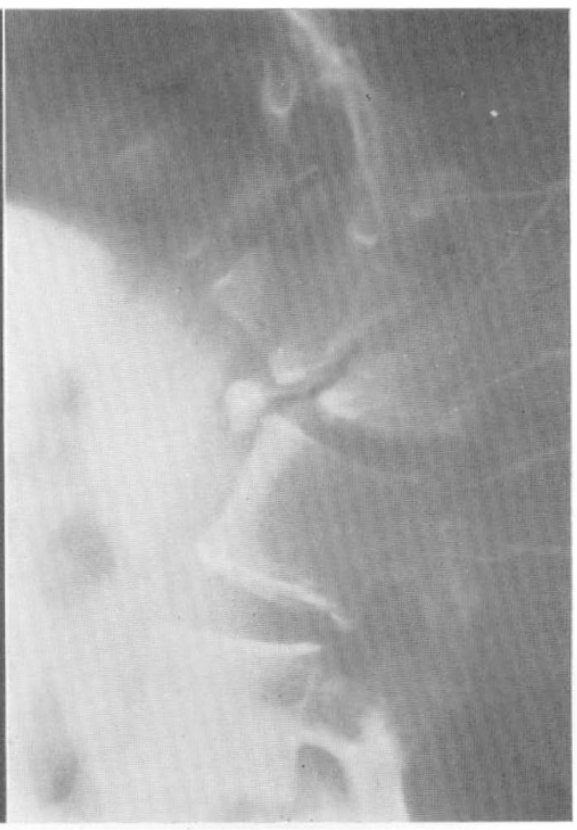

FIG. 8

Fig. 7.-The X-ray taken while this I9-year-old male patient was still on the Stryker frame following a laminectomy and spine fusion, Dr2-L2, without internal fixation. $\mathrm{He}$ had a complete cauda equina lesion.

Fig. 8.-One year later in spite of eight weeks on a Stryker frame, and continued use of a brace, the deformity had increased to 67 degrees. The density of the anterior portion of the body of L2 may represent avascular necrosis of bone. $\mathrm{He}$ continued to have back pain at the time of follow-up.

Skin problems are difficult to attribute to spine alignment abnormalities because it is well known that uicerations occur when no such mechanical defects are present. In two patients with these deformities, $\mathrm{AP} 65$, and lateral angulation I 5 degrees, and $\mathrm{AP}$ 55, Io degrees lateral deformities, we suspected that the deformities were factors in the production of ischial ulcers.

We were suspicious; but could not prove that a sacral ulcer was related to a 35 degree AP curve in one patient.

No patients in this group showed neurological deterioration related to the spinal deformity.

Lumbar Group (L2-L5). This group was limited to seven patients only. The average AP deformity was 52 degrees. Considering that the portion of the spine has a normal lordosis, the degree of deformity actually exceeded the measured angulation. 
Five patients had AP deformities, and three lateral. One patient actually had a 52 degree $\mathrm{AP}$ and a 32 degree lateral deformity.

None of the patients had postural sitting or standing problems. The lateral deformities did not affect balance.

Back pain was a major complaint of three of the seven patients, the pain persisted for four years in one patient and then disappeared. The pain has remained for eight years, and one year after injury in the remaining two patients. However, this pain has not been disabling. Surgery was recommended in one of the last two patients, but he refused it.

Progression of the spinal deformity occurred in two of five of the patients, both of whom had laminectomies and one also had a spine fusion at the time of laminectomy. The X-rays of these two patients suggest an avascular necrosis similar to those seen in the dorso-lumbar group (figs. 7 and 8).

None of the incomplete cauda equina lesions demonstrated any untoward effect related tc the persistent spine deformity. However, one cannot conclude from these observations that the neurological recovery was not in some way affected by the deformity, nor can one argue that it was significantly affected.

\section{CONCLUSIONS}

After reviewing 204 patients with paraplegia related to spinal fracture dislocations of the dorsal and lumbar spine, we found that AP deformities of 30 degrees or more did not significantly affect function in this group of patients. Sitting and standing function was not affected. Back pain was not a major complaint. Most patients developed solid spines spontaneously or following fusions. A small number of patients showed a persistent failure of fusion after internal fixation and bone grafting.

A small number of patients with fracture-dislocations in the dorso-lumbar or lumbar area showed progression of deformity in spite of spine fusion, Stryker frame immobilisation, bed rest, and braces. The X-rays suggest that the damaged vertebral bodies undergo avascular necrosis.

Our regimen of six weeks on the Stryker frame, followed by 6 to 12 additional weeks of back support with an appropriate brace did not prevent progress of deformity in a small number of patients, this time interval may be too short. However, even posterior spine fusion with or without internal fixation may be inadequate to prevent certain fractures to deform.

However, most of the fracture-dislocations in the dorso-lumbar or lumbar area do become solid over a period of several years. When they do, pain appears to subside and the deformity is not an obstacle to satisfactory function.

Back pain was not a major problem in this group of patients.

Furthermore, this study did not indicate that these major deformities caused deterioration of neurological function in the incomplete lesions.

The lateral spine deformities of Io degrees or more, and pelvic obliquity, may be very important as a cause of ischial ulcerations. Therefore more attention must be given to these lateral trunk deformities in paraplegics.

\section{REFERENCES}

Aufranc, O. E., Jobe, W. N. \& Bierbaum, B. (I968). Fracture of the Month \#86. F. Am. med. Assoc. 203, I 127.

Bedbrook, G. (I968). Personal Communication. 